\title{
ON SET CORRESPONDENCES INTO UNIFORMLY CONVEX BANACH SPACES ${ }^{1}$
}

\author{
DAVID SCHMEIDLER
}

\begin{abstract}
It is proved that the values of a set-valued set function, the total variation of which is an atomless finite measure, are conditionally convex.
\end{abstract}

Let $\Sigma$ be a nonempty $\sigma$-field of subsets of a set $S$. A (set) correspondence, say $\Gamma$, from $\Sigma$ to a Banach space $X$ maps, by definition, every element $E$ of $\Sigma$ to $\Gamma(E)$, a nonempty subset of $X$. In this paper $E, F, G$ denote elements of $\Sigma$ and $x, y, z$ denote elements of $X$. If $A$ and $B$ are nonempty subsets of $X$ then $A+B=\{x+y \mid x \in A$ and $y \in B\}$. The correspondence $\Gamma$ is additive if $\Gamma(E)+\Gamma(F)=\Gamma(E \cup F)+\Gamma(E \cap F)$ for all $E, F$ in $\Sigma$. The total variation (or simply variation) of $\Gamma$ is the extended real valued set function, say $v$, on $\Sigma$ defined as follows:

$$
\begin{aligned}
& v(E)=\sup \left\{\sum\left|x_{i}\right| \mid\left(x_{i}, E_{i}\right) \text { is a finite sequence in } X \times \Sigma,\right. \\
& \left.\left.\qquad E_{i}\right\} \text { is a partition of } E \text { and for all } i, x_{i} \in \Gamma\left(E_{i}\right)\right\} .
\end{aligned}
$$

This is a direct generalization of the notion of total variation for Banach valued measures. Our main result is:

THEOREM 1. Let $\Gamma$ be an additive correspondence from $\Sigma$ to a uniformly convex Banach space $X$. If the total variation of $\Gamma$ is an atomless finite measure on $\Sigma$ then the closure of $\Gamma(E)$ is convex for all $E$ in $\Sigma$. (In particular, if $\Gamma(E)$ is closed it is convex.)

This result is a generalization of Theorem 1.2 in [3]. The generalization consists of relaxing the finite dimensionality assumption on $X$ in [3]. Let us recall that $X$ is uniformly convex if for any two sequences $\left(x_{n}\right),\left(y_{n}\right)$ in the unit ball of $X,\left|x_{n}+y_{n}\right| \rightarrow 2$ implies $\left|x_{n}-y_{n}\right| \rightarrow 0$. The spaces $L^{p}$ and $L_{B}^{p}$ are uniformly convex for $1<p<\infty$ and $B$ a uniformly convex Banach space (see [2, §26.7.8]). The result holds, of course, if $X$ is uniformly normable, i.e. there is an equivalent norm which is uniformly convex. The reason is that atomlessness and finiteness of the total variation are

Presented to the Society, June 19, 1971; received by the editors April 21, 1971.

AMS 1970 subject classifications. Primary 26A45, 46G10.

1 The research for this paper was carried out with the support of National Science Foundation Grant GS-3274. 
invariant under equivalence of norms. It should be mentioned that another generalization of Theorem 1.2 in [3] was given in [1]. There the assumption of closedness was dispensed with. In the end of the paper an example is given where $\Gamma$ has nonconvex values because $X$ is not uniformly convex. Our next result is:

THEOREM 2. Under the conditions of Theorem 1 the closure of the range of $\Gamma$ is convex.

The range of the correspondence $\Gamma$ is the set $\bigcup_{E \in \Sigma} \Gamma(E)$ in $X$. In the finite dimensional case the conclusion is stronger (see [1], [3] and [4]). Convexity of the range of $\Gamma$ is a necessary tool in general equilibrium theory [4].

To simplify the proofs we start with several lemmas.

LEMMA 1. Let $\Gamma$ be an additive correspondence from $\Sigma$ to a Banach space $X$ and suppose that $v$, the total variation of $\Gamma$, is a finite measure on $\Sigma$. For $E$ in $\Sigma$ and $X$ in $\Gamma(E)$ we define

$u(x, E)=\sup \left\{\sum\left|x_{i}\right| \mid\left(x_{i}, E_{i}\right)\right.$ is a finite sequence in $X \times \Sigma$,

$\left\{E_{i}\right\}$ is a partition of $E, x_{i} \in \Gamma\left(E_{i}\right)$ for all $i$ and $\left.\sum x_{i}=x\right\}$.

Given $\varepsilon>0, \delta>0, \bar{E}$ in $\Sigma$ and $\bar{x}$ in $\Gamma(\bar{E})$ we have then a finite sequence $\left(\bar{x}_{i}, \bar{E}_{i}\right)$, say $i=0,1, \cdots, k$, in $X \times \Sigma$ so that $\left\{\bar{E}_{i}\right\}$ is a partition of $\bar{E}$, $\bar{x}_{i} \in \Gamma\left(\bar{E}_{i}\right)$ for all $i, \sum \bar{x}_{i}=\bar{x}, u\left(\bar{x}_{i}, \bar{E}_{i}\right)-\left|\bar{x}_{i}\right| \leqq \varepsilon v\left(\bar{E}_{i}\right)$ for $i=1, \cdots, k$, and $v\left(\bar{E}_{0}\right)<\delta$ (hence also $\left|\bar{x}_{0}\right|<\delta$ ).

Proof. First we observe that $u$ is superadditive and bounded by $v$. Superadditivity here means that for all $x, y, E, F$ such that $E \cap F=\varnothing$, $x \in \Gamma(E)$ and $y \in \Gamma(F)$ we have $u(x, E)+(u, F) \leqq u(x+y, E \cup F)$. Boundedness by $v$ means that for all $x, E$ with $x \in \Gamma(E)$ we have $u(x, E) \leqq v(E)$.

For any $\alpha>0$ we have, by the definition of $u(\bar{x}, \bar{E})$, a finite sequence $\left(x_{i}, F_{i}\right)$ so that $\left\{F_{i}\right\}$ is a partition of $\bar{E}, \sum_{i} x_{i}=\bar{x}, u(\bar{x}, \bar{E})-\sum_{i}\left|x_{i}\right| \leqq \alpha \varepsilon v(\bar{E})$ and for all $i, x_{i} \in \Gamma\left(F_{i}\right)$. Let $I$ be the subset of the indices $\{i\}$ such that for all $i$ in $I, u\left(x_{i}, F_{i}\right)-\left|x_{i}\right|>\varepsilon v\left(F_{i}\right)$. Then

$$
\begin{aligned}
\alpha \varepsilon v(\bar{E}) & \geqq u(\bar{x}, \bar{E})-\sum_{i}\left|x_{i}\right| \geqq \sum_{i}\left(u\left(x_{i}, F_{i}\right)-\left|x_{i}\right|\right) \\
& \geqq \sum_{i \in I}\left(u\left(x_{i}, F_{i}\right)-\left|x_{i}\right|\right)>\sum_{i \in I} \varepsilon v\left(F_{i}\right)=\varepsilon v\left(\bigcup_{i \in I} F_{i}\right) .
\end{aligned}
$$

(In addition to superadditivity of $u$ the inequality $u\left(x_{i}, F_{i}\right)-\left|x_{i}\right| \geqq 0$ was used.) To complete the proof of the lemma we define $\alpha$ by the equation $\delta=\alpha v(\bar{E})$, which implies that $\delta>v\left(\bigcup_{i \in I} F_{i}\right)$. Also we denote $\bigcup_{i \in I} F_{i}$ by $\bar{E}_{0}, \sum_{i \in I} x_{i}$ by $\bar{x}_{0}$, and for all $i$ not in $I$ let $\bar{E}_{i}=F_{i}$ and $\bar{x}_{i}=x_{i}$. Q.E.D. 
LemMa 2. Suppose, in addition to the assumptions of Lemma 1, that $v$ is atomless. Then for any $\delta>0$ and $(x, E)$ with $x \in \Gamma(E)$ we have $x_{1}$, $x_{2}, E_{1}, E_{2}$ with the following properties: $\left\{E_{1}, E_{2}\right\}$ is a partition of $E, x_{1}+$ $x_{2}=x, x_{1} \in \Gamma\left(E_{1}\right), x_{2} \in \Gamma\left(E_{2}\right)$ and ||$x_{1}|-| x_{2}||<\delta$.

Proof. By the atomlessness assumption there is a finite partition, say $\left\{F_{1}, \cdots, F_{k}\right\}$ of $E$ with $v\left(F_{i}\right)<\delta / 2$ for $i=1, \cdots, k$. Let $y_{i} \in \Gamma\left(F_{i}\right) ; i=$ $1, \cdots, k$ and $\sum y_{i}=x$ (such decomposition of $x$ exists because of the additivity of $\Gamma$ ). We have $\left|y_{i}\right|<\delta / 2$ for all $i$. Let $j$ be such that $\left|\sum_{i=1}^{j} y_{i}\right| \geqq$ $\left|\sum_{i=j+1}^{k} y_{i}\right|$ but $\left|\sum_{i=1}^{j-1} y_{i}\right| \leqq\left|\sum_{i=j}^{k} y_{i}\right|$ (we use the convention that summation over an empty set gives the origin of $X$ ). Then

$$
\begin{aligned}
0 & \leqq\left|\sum_{i=j}^{k} y_{i}\right|-\left|\sum_{i=1}^{j-1} y_{i}\right| \\
& \leqq\left(\left|\sum_{i=j+1}^{k} y_{i}\right|+\left|y_{j}\right|\right)-\left(\left|\sum_{i=1}^{j} y_{i}\right|-\left|y_{j}\right|\right) \leqq 2\left|y_{j}\right|<\delta .
\end{aligned}
$$

To complete the proof we define $x_{1}=\sum_{i=1}^{j-1} y_{i}, x_{2}=\sum_{i=j}^{k} y_{i}, E_{1}=\bigcup_{i=1}^{j-1} F_{i}$ and $E_{2}=\bigcup_{i=j}^{k} F_{i}$. Q.E.D.

Lemma 3. Suppose, in addition to the assumptions of Lemma 2, that $X$ is uniformly convex. Then for every $\delta>0$ there is $\varepsilon>0$ such that for any $x$, $y, z, E, F, G$ with $x \in \Gamma(E), y \in \Gamma(F), z \in \Gamma(G), x=y+z,\{F, G\}$ is $a$ partition of $E, \quad|| y|-| z||<\varepsilon v(E)$ and $u(x, E)-|x|<\varepsilon v(E)$ we have $|y-z| \leqq \delta v(E)$.

Proof. If the lemma does not hold then there is $\delta>0$ and sequences $\left(x_{i}\right),\left(y_{i}\right),\left(z_{i}\right),\left(E_{i}\right),\left(F_{i}\right),\left(G_{i}\right)$ such that for all $i, x_{i} \in \Gamma\left(E_{i}\right), y_{i} \in \Gamma\left(F_{i}\right)$, $z_{i} \in \Gamma\left(G_{i}\right), x_{i}=y_{i}+z_{i},\left\{F_{i}, G_{i}\right\}$ is a partition of $E_{i}$,

$$
\begin{array}{r}
|| y_{i}|-| z_{i}||<v\left(E_{i}\right) / i, \\
u\left(x_{i}, E_{i}\right)-\left|x_{i}\right|<v\left(E_{i}\right) / i
\end{array}
$$

and

$$
\left|y_{i}-z_{i}\right|>\delta v\left(E_{i}\right) .
$$

We have also, for all $i, 0 \leqq\left|y_{i}\right|+\left|z_{i}\right|-\left|x_{i}\right| \leqq u\left(x_{i}, E_{i}\right)-\left|x_{i}\right|<v\left(E_{i}\right) / i$. For all $i$, we assume, without loss of generality, that $\left|y_{i}\right| \geqq\left|z_{i}\right|$ and we define $x_{i}^{\prime}$, $y_{i}^{\prime}$ and $z_{i}^{\prime}$ by the equations: $x_{i}^{\prime}\left|y_{i}\right|=x_{i}, y_{i}^{\prime}\left|y_{i}\right|=y_{i}$ and $z_{i}^{\prime}\left|y_{i}\right|=z_{i}$. For every $i$,

$$
\delta v\left(E_{i}\right)<\left|y_{i}-z_{i}\right| \leqq\left|y_{i}\right|+\left|z_{i}\right| \leqq\left|y_{i}\right|+\left|y_{i}\right|,
$$

hence $\left|y_{i}\right|>\left(\frac{1}{2}\right) \delta v\left(E_{i}\right)$ or $\left|y_{i}\right| / v\left(E_{i}\right)>\delta / 2$. On the other hand, $\left|y_{i}\right| / v\left(E_{i}\right) \leqq 1$. 
Thus, $\left|z_{i}^{\prime}\right| \leqq\left|y_{i}^{\prime}\right|=1$ and

$$
\begin{aligned}
\left|x_{i}^{\prime}\right| & \geqq\left|y_{i}^{\prime}\right|+\left|z_{i}^{\prime}\right|-v\left(E_{i}\right) / i\left|y_{i}\right| \\
& \geqq\left|y_{i}^{\prime}\right|+\left|y_{i}^{\prime}\right|-v\left(E_{i}\right) / i\left|y_{i}\right|-v\left(E_{i}\right) / i\left|y_{i}\right| \\
& =2-(2 / i)\left(v\left(E_{i}\right) /\left|y_{i}\right|\right) \geqq 2-(2 / i)(2 / \delta),
\end{aligned}
$$

i.e. $\left|x_{i}^{\prime}\right| \rightarrow 2$ when $i \rightarrow \infty$. By the uniform convexity of $X$ we have $\left|y_{i}^{\prime}-z_{i}^{\prime}\right| \rightarrow 0$ when $i \rightarrow \infty$. However $\left|y_{i}^{\prime}-z_{i}^{\prime}\right|>\delta v\left(E_{i}\right) /\left|y_{i}\right| \geqq \delta$, a contradiction. Q.E.D.

Proof OF THEOREM 1. In order to show the convexity of the closure of $\Gamma(\bar{E})$ it is sufficient to prove that for any $\bar{x}, \bar{y}$ in $\Gamma(\bar{E})$ and every $\alpha>0$ there is $\bar{z}$ in $\Gamma(\bar{E})$ with $|(\bar{x}+\bar{y}) / 2-\bar{z}|<\alpha$.

Let $X \times X$ be the Banach space with norm $|(x, y)|=\left(|x|^{2}+|y|^{2}\right)^{1 / 2}$. This norm is uniformly convex because the norm of $X$ is. For every $E$ in $\Sigma$ we define $\Gamma^{\prime}(E)=\Gamma(E) \times \Gamma(E) \subset X \times X$. Let $v^{\prime}$ be the total variation of $\Gamma^{\prime}$; then $v^{\prime}$ is a finite atomless measure. We have, in fact, that $v^{\prime}(E)=2^{1 / 2} v(E)$ for all $E$ in $\Sigma$. We will apply Lemmas 1,2 and 3 to $\Gamma^{\prime}$.

Let $\bar{\varepsilon}$ be the $\varepsilon>0$ corresponding to $\delta$ of Lemma 3 where $\delta>0$ is defined by $\alpha=4 \delta v^{\prime}(\bar{E})$. (We rule out the uninteresting case where $v^{\prime}(\bar{E})=0$ because then $v(\bar{E})=0$ which in turn implies that $\bar{x}=\bar{y}=0$.) Now we apply Lemma 1 to $\Gamma^{\prime}(\bar{E})$ with $\varepsilon=\bar{\varepsilon}$ and $\delta=\alpha / 4$. Hence we have a partition $\left\{E_{0}, E_{1}, \cdots, E_{k}\right\}$ of $\bar{E}$ and vectors $\left(x_{i}, x_{i}^{\prime}\right)_{i=0}^{k}$ in $X \times X$ so that $\left(x_{i}, x_{i}^{\prime}\right) \in \Gamma^{\prime}\left(E_{i}\right)$ for all $i$, $\sum x_{i}=\bar{x}, \sum x_{i}^{\prime}=\bar{y},\left|\left(x_{0}, x_{0}^{\prime}\right)\right| \leqq v^{\prime}\left(E_{0}\right)<\alpha / 4$ and $u\left(\left(x_{i}, x_{i}^{\prime}\right), E_{i}\right)-\left|\left(x_{i}, x_{i}^{\prime}\right)\right| \leqq$ $\bar{\varepsilon} v^{\prime}\left(E_{i}\right)$ for $i=1, \cdots, k$. By Lemma 2 , for $i=1, \cdots, k$, there is a partition, say $\left\{F_{i}, G_{i}\right\}$, of $E_{i}$ and there are vectors $\left(y_{i}, y_{i}^{\prime}\right)$ in $\Gamma^{\prime}\left(F_{i}\right)$ and $\left(z_{i}, z_{i}^{\prime}\right)$ in $\Gamma^{\prime}\left(G_{i}\right)$ so that $\left(y_{i}, y_{i}^{\prime}\right)+\left(z_{i}, z_{i}^{\prime}\right)=\left(x_{i}, x_{i}^{\prime}\right)$ and ||$\left(y_{i}, y_{i}^{\prime}\right)|-|\left(z_{i}, z_{i}^{\prime}\right)|| \leqq$ $\bar{\varepsilon} v\left(E_{i}\right)$. By Lemma 3, $\left|\left(y_{i}, y_{i}^{\prime}\right)-\left(z_{i}, z_{i}^{\prime}\right)\right| \leqq \delta v^{\prime}\left(E_{i}\right)=\alpha v^{\prime}\left(E_{i}\right) / 4 v^{\prime}(\bar{E})$. Let $\bar{z}=x_{0}+\sum_{i=1}^{k} y_{i}+\sum_{i=1}^{k} z_{i}^{\prime}$. Then

$$
\bar{z} \in \Gamma\left(E_{0} \cup\left(\bigcup_{i=1}^{k} F_{i}\right) \cup\left(\bigcup_{i=1}^{k} G_{i}\right)\right)=\Gamma(\bar{E}) .
$$

To complete the proof we show that $|\bar{x}+\bar{y}-2 \bar{z}|<2 \alpha$. Indeed

$$
\begin{aligned}
\mid \bar{x}+ & \bar{y}-2 \bar{z} \mid \\
& =\left|x_{0}+\sum_{i=1}^{k}\left(y_{i}+z_{i}\right)+x_{0}^{\prime}+\sum_{i=1}^{k}\left(y_{i}^{\prime}+z_{i}^{\prime}\right)-2 x_{0}-2 \sum_{i=1}^{k} y_{i}-2 \sum_{i=1}^{k} z_{i}^{\prime}\right| \\
& \leqq\left|x_{0}-x_{0}^{\prime}\right|+\sum_{i=1}^{k}\left|y_{i}-z_{i}\right|+\sum_{i=1}^{k}\left|y_{i}^{\prime}-z_{i}^{\prime}\right| \\
& \leqq\left|x_{0}\right|+\left|x_{0}^{\prime}\right|+2 \delta \sum_{i=1}^{k} v^{\prime}\left(E_{i}\right) \\
& \leqq \alpha / 4+\alpha / 4+\alpha / 2=\alpha . \quad \text { Q.E.D. }
\end{aligned}
$$

ProOF OF THEOREM 2. In order to prove the convexity of the closure of the range of $\Gamma$ it is sufficient to show that for every $\bar{x}$ and $\bar{y}$ in the range of $\Gamma$ and every $\alpha>0$ there is $\bar{z}$ in the range of $\Gamma$ with $|(\bar{x}+\bar{y}) / 2-\bar{z}|<\alpha$. Let $\bar{x}$ 
be in $\Gamma(\bar{E})$ and $\bar{y}$ in $\Gamma(\bar{F})$ for some $\bar{E}$ and $F$ in $\Sigma$. Then $x=\bar{x}_{1}+\bar{x}_{2}$ and $\bar{y}=\bar{y}_{2}+\bar{y}_{3}$ for some $\bar{x}_{1}$ in $\Gamma(\bar{E} \backslash F), \bar{x}_{2}$ and $\bar{y}_{2}$ in $\Gamma(\bar{E} \cap \bar{F})$ and $\bar{y}_{3}$ in $\Gamma(\bar{F} \mid \bar{E})$. By Theorem 1 there is $\bar{z}_{2}$ in $\Gamma(\bar{E} \cap \bar{F})$ with $\left|\left(\bar{x}_{2}+\bar{y}_{2}\right) / 2-\bar{z}_{2}\right|<\alpha / 3$. To complete the proof we show that there are $\bar{G}$ in $\Sigma, \bar{G} \subset(\bar{E} \backslash \bar{F})$ and $\bar{z}_{1}$ in $\Gamma(\bar{G})$ with $\left|\bar{x}_{1} / 2-\bar{z}_{1}\right|<\alpha / 3$.

We apply Lemma 1 to $\bar{x}_{1}$ in $\Gamma(\bar{E} \backslash \bar{F})$ with $\delta=\alpha / 6$ and $\varepsilon$ defined by Lemma 3 where the $\delta$ of Lemma 3 is $\alpha /(6 v(\bar{E} \backslash \bar{F}))$. By Lemma 1 there is a partition $\left\{E_{0}, E_{1}, \cdots, E_{k}\right\}$ of $\bar{E} \backslash \bar{F}$ and vectors $x_{i}$ in $\Gamma\left(E_{i}\right)$ with $\sum x_{i}=\bar{x}_{1}$, $\left|x_{0}\right| \leqq v\left(E_{0}\right)<\alpha / 6$ and $u\left(x_{i}, E_{i}\right)-\left|x_{i}\right| \leqq \varepsilon v\left(E_{i}\right)$ for $i=1, \cdots, k$. By Lemma 2 we have, for $i=1, \cdots, k,\left\{F_{i}, G_{i}\right\}$ a partition of $E_{i}, y_{i} \in \Gamma\left(F_{i}\right), z_{i} \in$ $\Gamma\left(G_{i}\right), y_{i}+z_{i}=x_{i}$ and ||$y_{i}|-| z_{i}||<\varepsilon v\left(E_{i}\right)$. By Lemma 3, $\left|y_{i}-z_{i}\right|<$ $\left(\alpha v\left(E_{i}\right)\right) /(6 v(\bar{E} \backslash \bar{F}))$. Let $\bar{z}_{1}=\sum_{i=1}^{k} z_{i}$. Then

$$
\begin{aligned}
\left|\bar{x}_{1}-2 \bar{z}_{1}\right| & =\left|\sum_{i=0}^{k} x_{i}-2 \sum_{i=1}^{k} z_{i}\right|=\left|x_{0}+\sum_{i=1}^{k}\left(y_{i}+z_{i}\right)-2 \sum_{i=1}^{k} z_{i}\right| \\
& \leqq\left|x_{0}\right|+\sum_{i=1}^{k}\left|y_{i}-z_{i}\right| \leqq \frac{\alpha}{6}+\frac{\left(\alpha \sum_{i=1}^{k} v\left(E_{i}\right)\right)}{(6 v(\bar{E} \backslash \bar{F}))} \leqq \frac{\alpha}{3} \text {. Q.E.D. }
\end{aligned}
$$

A counterexample. Let $S$ be the unit interval, $\Sigma$ its Lebesgue measurable subsets, $\lambda$ a Lebesgue measure on $\Sigma$ and $X=L^{1}(S, \Sigma, \lambda)$. We define $\Gamma(E)=\left\{x_{F} \in X \mid F \subset E\right.$ and $\left.F \in \Sigma\right\}$ ( $x_{F}$ is the characteristic function of the set $F$ ). The variation of $\Gamma$ is $\lambda$ which is atomless and a finite measure and the values of $\Gamma$ on $\Sigma$ and its range are closed in $X$. But $\Gamma(E)$ is not convex for any nonnull $E$ and the range of $\Gamma$ is $\Gamma(S)$. If we consider $X$ as $L^{2}(S, \Sigma, \lambda)$ then $\Gamma$ is well defined but the variation of every nonnull set is infinite.

\section{REFERENCES}

1. Z. Artstein, Set-valued measures, Research Program in Game Theory and Mathematical Economics, R.M. \#66, May 1971, Department of Mathematics, The Hebrew University of Jerusalem; previous version: R.M. \#62, July 1970.

2. G. Köthe, Topological vector spaces. Vol. I, Die Grundlehren der math. Wissenschaften, Band 159, Springer-Verlag, New York, 1969. MR 40 \#1750.

3. D. Schmeidler, "Convexity and compactness in countably additive correspondences," Differential games and related topics (edited by H. W. Kuhn and G. P. Szego), North-Holland, Amsterdam, 1971. See also: Center for Operations Research and Econometrics, D.P. \#7019, May 1970, Université Catholique de Louvain.

4. K. Vind, Edgeworth Allocations in an exchange economy with many traders, Internat. Economic Rev. 5 (1964), 165-177.

Center for Research in Management Science, University of California, Berkeley, California 94720

Current address: Department of Statistics, Tel-Aviv University, Tel-Aviv, Israel 